\title{
Magnolol may contribute to barrier function improvement on imiquimod-induced psoriasis-like dermatitis animal model via the downregulation of interleukin-23
}

\author{
JIUN-WEN GUO ${ }^{1,2}$, YU-PIN CHENG ${ }^{3}$, CHIH-YI LIU ${ }^{4}$, HAW-YUEH THONG ${ }^{5}$, \\ YANG LO ${ }^{3}$, CHEN-YU WU ${ }^{3}$ and SHIOU-HWA JEE ${ }^{3}$ \\ ${ }^{1}$ Department of Medical Research, Cathay General Hospital, Taipei 10630; \\ ${ }^{2}$ College of Medicine, Fu Jen Catholic University, New Taipei City 24205; \\ ${ }^{3}$ Department of Dermatology, Cathay General Hospital, Taipei 10630; \\ ${ }^{4}$ Division of Pathology, Sijhih Cathay General Hospital, New Taipei City 22174; \\ ${ }^{5}$ Department of Dermatology, Shin-Kong Wu Ho-Su Memorial Hospital, Taipei 11101, Taiwan, R.O.C.
}

Received April 22, 2020; Accepted September 16, 2020

DOI: $10.3892 /$ etm.2021.9876

\begin{abstract}
Psoriasis is a chronic, recurrent, immune-mediated disease involving the skin and joints. Epidermal hyperproliferation, abnormal keratinocyte differentiation, angiogenesis with blood vessel dilatation, and excess T helper type-1 (Th-1) and Th-17 cell infiltration are the main histopathological features of psoriasis. Magnolol is a polyphenolic compound that exerts its biological properties through a variety of mechanisms such as the NF- $\mathrm{BB} / \mathrm{MAPK}, \mathrm{Nrf} 2 / \mathrm{HO}-1$ and PI3K/Akt pathways. Magnolol has been demonstrated to exert a number of therapeutic effects on dermatological processes, including acting as an anti-inflammation, antiproliferation and antioxidation agent. However, few studies have been published on the effect of magnolol on psoriasis. Therefore, the present study aimed to elucidate the mechanism of action of magnolol on psoriasis. $\mathrm{BALB} / \mathrm{c}$ mice were treated topically with imiquimod (IMQ) to induce psoriasis-like dermatitis, and were randomly assigned to the control, vehicle control, low- and high-dose magnolol, and $0.25 \%$ desoximetasone ointment treatment groups in order to investigate skin barrier function, any changes in the levels of cytokines and for the histological assessment. High doses of magnolol were indicated to be able to improve the barrier function following IMQ-induced barrier disruption. Magnolol activated peroxisome proliferator-activated receptor- $\gamma$, and
\end{abstract}

Correspondence to: Dr Shiou-Hwa Jee, Department of Dermatology, Cathay General Hospital, Section 4, 280 Ren-Ai Road, Taipei 10630, Taiwan, R.O.C.

E-mail: shiouhwa@gmail.com

Abbreviations: PPAR- $\gamma$, peroxisome proliferator-activated receptor- $\gamma$; IMQ, imiquimod; DXM, 0.25\% desoximetasone ointment; EtOH, ethanol; TEWL, transepidermal water loss; DAB, diaminobenzidine

Key words: interleukin-17, interleukin-23, psoriasis, magnolol, imiquimod, skin barrier also significantly inhibited the protein expression of interleukin (IL)-23, IL-1 $\beta$, IL-6, tumor necrosis factor- $\alpha$ and interferon- $\gamma$. However, administering a high dose of magnolol did not lead to any improvement in the clinical and pathological features of the psoriasis severity Taken together, these results demonstrated that downregulation of IL-23 may contribute to barrier function improvement in a psoriatic skin model.

\section{Introduction}

A healthy skin barrier can be attributed to well-differentiated corneocytes, correctly arranged extracellular lipid bilayers, balanced activities of antimicrobial peptides and enzymes, and a physiologically weak acidic $\mathrm{pH}$ environment on the skin surface (1). Inflammatory skin conditions, such as psoriasis and atopic dermatitis, present with impaired skin barrier function (1). Psoriasis is a chronic, recurrent immune-mediated disease and affects people of all ages, most commonly in individuals aged between 15-30 years old (2-4). Epidermal hyperplasia, acanthosis, hyperparakeratosis, angiogenesis with blood vessel dilatation and excess $\mathrm{T}$ helper type-1 (Th-1) and Th-17 lymphocyte infiltration are the main histopathological features of psoriasis (2-5). Furthermore, the interleukin (IL)-23/IL-17 axis model for psoriasis proposes that IL-23 activates Th17 lymphocytes, resulting in the subsequent release of proinflammatory cytokines, including IL-17, leading to the psoriatic phenotype $(5,6)$. Although genetic, immunological and environmental factors have been proposed as being the cause of this condition, the exact cause of psoriasis has yet to be elucidated, and the mechanisms underlying psoriasis continue to be poorly understood $(3,4,7)$.

Magnolol (5,5'-diallyi-2,2'-dihydroxy biphenyl; $\mathrm{C}_{18} \mathrm{H}_{18} \mathrm{O}_{2}$, $\mathrm{MW}=266.33 \mathrm{Da})$ is one of the major active polyphenolic ingredients isolated from Magnolia officinalis (known as houpu magnolia) $(8,9)$. The considerable efficacy of magnolol has been confirmed through an assessment of its anti-inflammatory, antiproliferative, anti-photoaging and anti-free radical activity $(8,10-14)$. Magnolol has also been 
indicated to be an agonist of peroxisome proliferator-activated receptor- $\gamma$ (PPAR- $\gamma$ ) (15-17). The key functions of PPAR- $\gamma$ in the epidermis include maintenance of skin barrier homeostasis, regulation of the stratum corneum surface $\mathrm{pH}$ and water-holding capacity, controlling cell differentiation and responding to inflammatory responses via PPAR- $\gamma$ activation, thus resulting in increased cell survival and reduced apoptosis in UV-induced damage studies $(18,19)$. However, the effect of magnolol on psoriasis has been less well reported, although it may be hypothesized that magnolol could contribute towards permeability barrier homeostasis via PPAR $-\gamma$ in psoriatic skin. Therefore, the present study aimed to investigate the therapeutic effects, as well as the effect on the skin barrier, of magnolol on an imiquimod (IMQ)-induced psoriatic-like dermatitis model. The underlying mechanisms governing this interaction were also investigated.

\section{Materials and methods}

Materials. Magnolol was purchased from Merck KGaA. Esperson (0.25\% Desoximetasone ointment) was purchased form Sanofi S.A. All other chemicals were of analytical grade.

Animals. A total of 15 male BALB/c mice (8-12 weeks old; purchased from National Laboratory Animal Center, Tainan, Taiwan), weighing $22 \pm 2 \mathrm{~g}$, were housed under standard laboratory conditions with sufficient food and water that was accessible at all times, as well as with minimized handling, odors, noises and vibrations in the Laboratory Animal Center of the Cathay General Hospital (12 h light/dark cycles and $24 \pm 2^{\circ} \mathrm{C}$ ambient temperature). A total of three mice were placed in each group. The duration of the experiment was 11 days. The animal health and behavior were monitored every day via body weight and food intake measurement. All animal experiments were performed and approved by the Institutional Animal Care and Use Committee (IACUC) of Cathay General Hospital (IACUC registration no. 107-028). During the experimental period, each animal was housed in a separate cage with wooden bar toys, complying with the IACUC regulations. To minimize distress during hair shaving, mice were placed in the induction anesthesia chamber $(10 \times 10 \times 20 \mathrm{~cm})$ with $4 \%$ isoflurane in oxygen (flow rate $=0.51 / \mathrm{min}$ ), followed by $2 \%$ isoflurane in oxygen (flow rate $=0.2 \mathrm{l} / \mathrm{min}$ ) for maintenance of anesthesia via a facemask. During the experimental period, the mice were euthanized via excessive isoflurane exposure followed by cervical dislocation to confirm successful euthanasia as a humane endpoint for this study, when cachexia led to a body-weight loss of $10 \%$ or more. No mice suffered spontaneous mortality or were euthanized because of a body-weight loss of $10 \%$ or more during the experiment. For euthanasia, mice were placed in the induction anesthesia chamber with $5 \%$ isoflurane in oxygen (flow rate $=0.5 \mathrm{l} / \mathrm{min}$ ) exposure continued for at least $1 \mathrm{~min}$ after respiratory arrest, followed by cervical dislocation to confirm successful euthanasia. All animals were sacrificed on day 11 at the end of the experiment to obtain the skin samples for further investigation.

Establishment of the IMQ-induced psoriasis-like skin animal model. Psoriasiform dermatitis was induced in mice following a widely used protocol (20-23) through the topical application of a dose of $62.5 \mathrm{mg} 5 \%$ Aldara IMQ cream (3M Pharmaceuticals) on the shaved dorsal skin for six consecutive days, once daily, prior to the experimental period (days 0-6) (20-23).

Experimental protocols. A total of 18 mice were used in the present study. Three untreated (normal) mice were used as negative control specimens for morphology, PPAR- $\gamma$ and cytokine array studies. For the barrier function study, 15 mice were randomly assigned to the following groups: i) The control group (only induced by IMQ); ii) vehicle group, treated with ethanol (EtOH; IMQ-induced plus EtOH treatment); iii) low-dose magnolol group (IMQ-induced, $100 \mu \mathrm{g} / \mathrm{ml}$ magnolol dissolved in EtOH treatment); iv) high-dose magnolol group (IMQ-induced, $300 \mu \mathrm{g} / \mathrm{ml}$ magnolol dissolved in EtOH treatment) and v) $0.25 \%$ desoximetasone ointment (DXM) group [IMQ-induced plus Esperson (0.25\% Desoximetasone ointment; Sanofi S.A.) treatment as a positive control]. Following the successful induction of the psoriasiform skin, the mice continued to receive IMQ application, followed by their respective treatments until day 11 . In the treatment phase (days 6-11), 3-4 h post-IMQ application, mice were treated once daily with $100 \mu \mathrm{l}$ magnolol, EtOH solution or $60 \mathrm{mg}$ DXM on the dorsal skin.

Assessment of barrier functions. Barrier function parameters, including transepidermal water loss (TEWL), skin hydration and erythema values, were measured on the dorsal surface of mice prior to the application of drugs on day 0 (used as normal barrier functions value baseline), and subsequently on days 6 and 11 using an MPA-II system equipped with Tewameter TM300, Corneometer CM825 and Mexmeter MX18 probes (Courage and Khazaka Electronic $\mathrm{GmbH}$ ).

Collection of skin specimens. Three untreated mice were sacrificed $48 \mathrm{~h}$ after hair shaving, and the specimens served as negative controls for inflammatory cytokine analysis. Treated (barrier function study) mice were sacrificed on day 11 after the final barrier function assessment. Full-thickness mouse skin was separated into two samples for histological staining.

Immunohistochemical staining for PPAR- $\gamma$. Skin specimens were fixed in $10 \%$ formalin solution and embedded in paraffin at $4{ }^{\circ} \mathrm{C}$ overnight. Sections of $5 \mu \mathrm{m}$ thickness were cut and stained with primary antibodies against PPAR- $\gamma$ (1:25; cat. no.GTX19481; Rabbit origin; GeneTex, Inc.) using a Ventana BenchMark XT automated stainer (Ventana Medical Systems, Inc.). Samples were incubated with the PPAR- $\gamma$ primary antibody (1:25; cat. no. GTX19481; Rabbit origin; GeneTex, Inc.) in universal ready to use blocking reagent (cat. no. 760-050; Ventana Medical Systems Inc.) for $60 \mathrm{~min}$ at $37^{\circ} \mathrm{C}$, and then the incubation was continued overnight at $4^{\circ} \mathrm{C}$. Subsequently, the samples were incubated with the universal mouse and rabbit ready to use secondary biotinylated antibody using an ultraView Universal DAB ready to use Detection kit (cat. no. 760-500; Ventana Medical Systems Inc.) for $1 \mathrm{~h}$ at room temperature. The levels of diaminobenzidine (DAB) were subsequently visualized. Samples were then counterstained with hematoxylin for $4 \mathrm{~min}$ at $37^{\circ} \mathrm{C}$, and examined under a light microscope at a magnification of x200 (BX41; Olympus Corporation). 

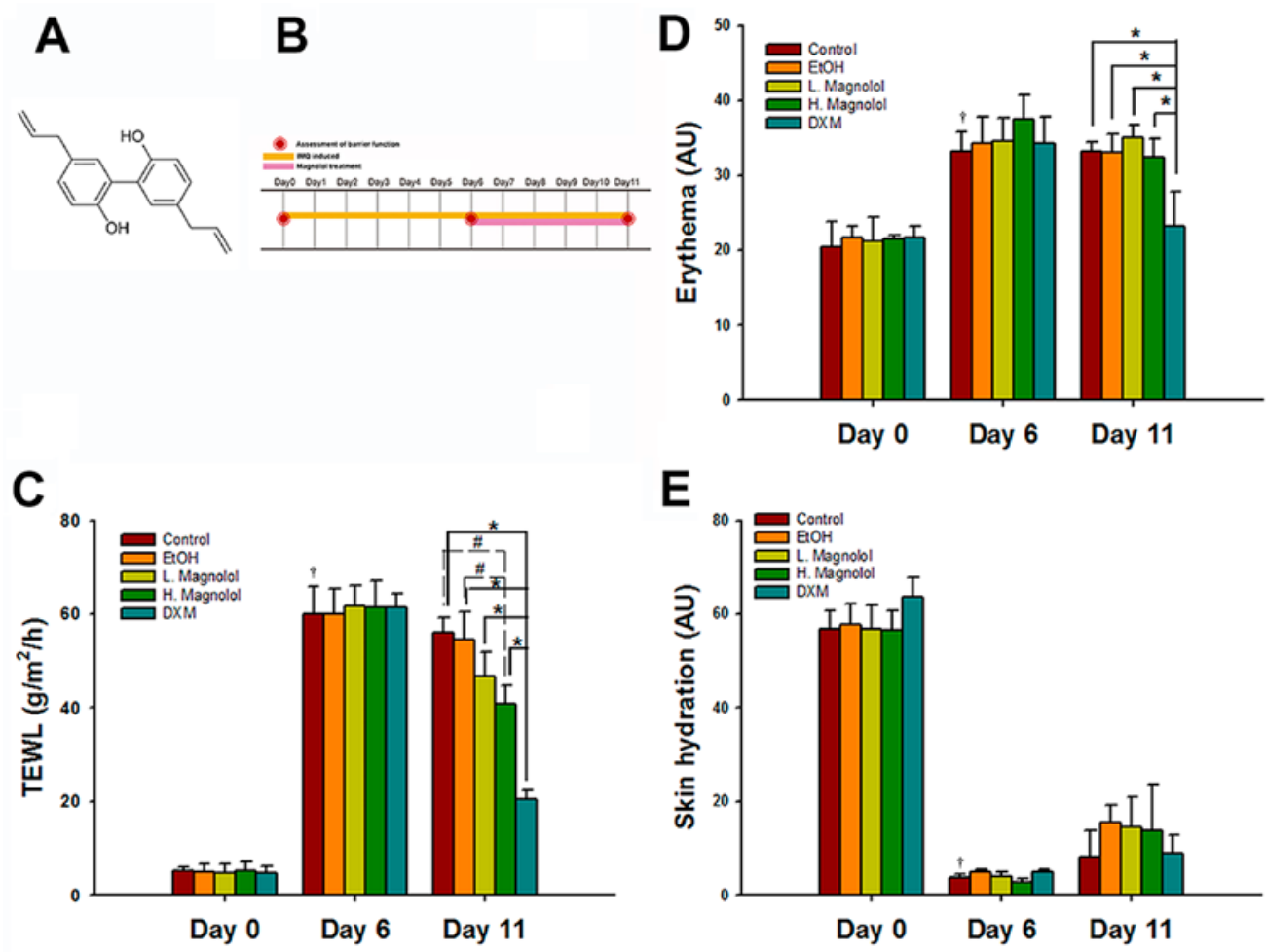

Figure 1. Magnolol improves the barrier function in IMQ-induced psoriasis-like dermatitis. (A) Chemical structure of magnolol. (B) Study design. Mice were administered a daily topical application of a dose of $62.5 \mathrm{mg}$ Aldara IMQ cream (5\%) on the shaved dorsal skin for 6 consecutive days (days 0-6). After the successful induction of psoriasiform skin, the mice continued to receive IMQ until day 11. After 3-4 h IMQ application, the mice subsequently received $100 \mu \mathrm{l}$ either magnolol, EtOH solution or $60 \mathrm{mg}$ DXM on the dorsal skin once daily (days 6-11). (C) TEWL, (D) skin hydration and (E) erythema values were measured on the dorsal surface of mice before the applications of drugs on day 0 , and then monitored on days 6 and 11 . (mean $\pm S D ; n=3)$. ${ }^{\dagger} \mathrm{P}<0.05$, compared with Day 0 and Day 6 in control group; ${ }^{\#} \mathrm{P}<0.05$ and ${ }^{*} \mathrm{P}<0.05$ (using two way mixed ANOVA, post hoc Bonferroni's test). IMQ, imiquimod; TEWL, transepidermal water loss; EtOH, ethanol, DMX, $0.25 \%$ desoximetasone ointment.

Immuno-intensity counting. To objectively evaluate the immunostaining results, the slides were scanned using a slide scanner (Pannoramic DESK II DW; 3DHISTECH Ltd.) at a magnification of x200. CellQuant and PatternQuant computer counting software (version 2.4.0) were used (all, 3DHISTECH Kft.). PatternQuant was programmed to recognize the regions of interest, and CellQuant was used to evaluate the $\mathrm{H}$-Score. Each skin tissue was assigned an annotation, which was $1 \mathrm{~mm}$ wide and covered the whole thickness of the skin to the muscle layer. The H-score was defined in terms of its immune-intensity, and this was then multiplied by the staining percentage, providing a range of values from 0-300. The immuno-intensity was recorded as being 0 for no staining, 1 for faint staining, 2 for moderate staining, and 3 for intense staining, whereas the staining percentage was recorded from $0-100 \%$. The immune-intensity and staining percentages were both determined using computer counting, as calculated by CellQuant, and counting was only permitted within the regions of interest recognized by PatternQuant.

Determination of the inflammatory cytokine protein levels via multiplex cytokines bead array. Studies have reported that IMQ-induced psoriasis-like skin elicits either the protein or mRNA expression of IL-17, IL-23, IL-1 $\beta$, IL-6, tumor necrosis factor- $\alpha$ (TNF- $\alpha)$, and interferon- $\gamma$ (IFN- $\gamma$ ) in mice, and successful anti-psoriatic interventions should therefore inhibit the aforementioned cytokine expression $(5,24-26)$. Therefore, in the current study, proteins were extracted from whole skin of 6 groups in order to determine the levels of IL-17A, IL-23,
IL-1 $\beta$, IL-6, TNF- $\alpha$ and IFN- $\gamma$ using the LEGENDplex ${ }^{\mathrm{TM}}$ multiplex cytokines bead array kit (mouse inflammation panel; BioLegend, Inc.). Aliquots $(50 \mu \mathrm{l})$ of protein samples were incubated with labeled microbeads for $2 \mathrm{~h}$ at room temperature, and subsequently, the concentration of each cytokine was determined using flow cytometry (using an Accuri C6 flow cytometer; BD Biosciences) according to the manufacturer's instructions. The concentration of each cytokine was then determined based on a known standard curve using LEGENDplex ${ }^{\mathrm{TM}}$ data analysis software version 8.0 (VigeneTech Inc.) (23).

Light microscopy. Skin samples were fixed in $10 \%$ formalin at $4^{\circ} \mathrm{C}$ overnight. Sample sections ( $5 \mu \mathrm{m}$-thick) were cut, stained with hematoxylin for $8 \mathrm{~min}$ and then eosin for $30 \mathrm{sec}$ both at room temperature (H\&E), and examined under a light microscope at a magnification of x200 (BX41; Olympus Corporation).

Statistical analysis. Two-way mixed ANOVA followed by Bonferroni's post hoc test was performed using SPSS 20 software (IBM Corp.). All bar charts are presented as the mean \pm standard deviation using Sigmaplot 10.0 software (Systat Software, Inc.). P $<0.05$ was considered to indicate a statistically significant difference.

\section{Results}

Magnolol improves the barrier function of IMQ-induced psoriasis-like dermatitis. The chemical structure of magnolol 


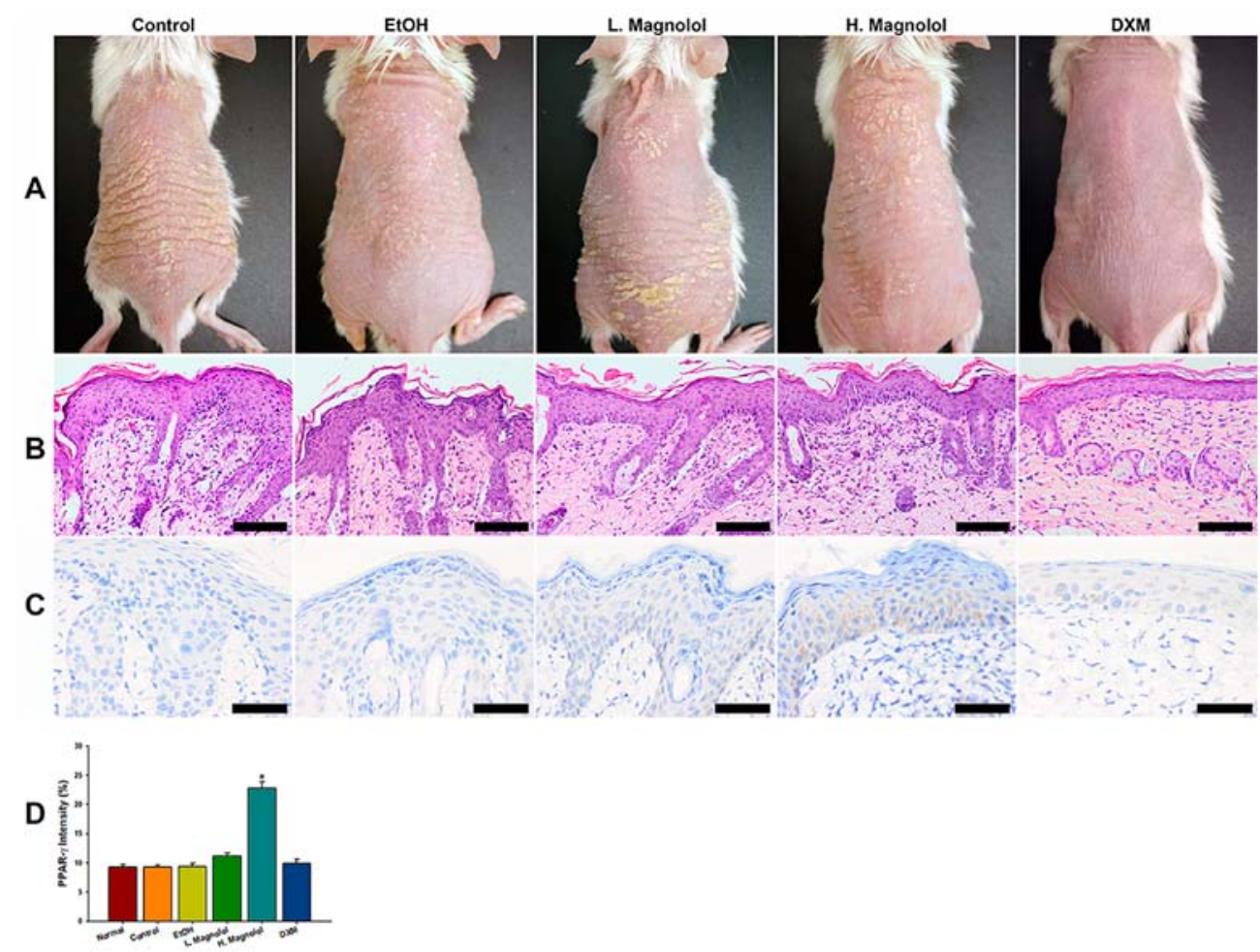

Figure 2. DXM inhibits hyperproliferation of keratinocytes. (A) Morphology of all the groups, revealing the presence of scales, erythema and dry skin. (B) Histopathological staining using H\&E revealed epidermal acathotic hyperplasia, abnormal keratinocyte differentiation, superficial dermal capillary dilatation and infiltration of various inflammatory cell types in all groups. However, the Esperson (DMX) treatment group showed much better gross and pathological features of severity index compared with all the other groups. (C) Magnolol activates PPAR- $\gamma$ protein expression. The high-dose magnolol group showed positive staining of PPAR- $\gamma$. (D) The immuno-intensity of PPAR- $\gamma$. The high-dose magnolol treatment group revealed a higher intensity of PPAR- $\gamma$ compared with all the other groups. Scale bar, $50 \mu \mathrm{m}$. Yellow arrow head indicated neutrophil cell. (mean \pm standard deviation, $\mathrm{n}=3$ ); ${ }^{*} \mathrm{P}<0.05$ (using two-way mixed ANOVA, post hoc Bonferroni's test). H\&E, hematoxylin and eosin; PPAR- $\gamma$, peroxisome proliferator-activated receptor- $\gamma$; DMX, $0.25 \%$ desoximetasone ointment.

and the study design are presented in Fig. 1A and B, respectively. The barrier function measurement values of the control group on day 0 represented the normal baseline values (TEWL, $7.39 \pm 1.20 \mathrm{~g} / \mathrm{m}^{2} / \mathrm{h}$; skin hydration, $58.25 \pm 5.84$ arbitrary units (AU); and erythema, 16.80 $\pm 1.74 \mathrm{AU})$. Compared with the values on day 0 (as normal baseline), the TEWL $\left(40.79 \pm 8.05 \mathrm{~g} / \mathrm{m}^{2} / \mathrm{h}\right)$ and erythema $(31.23 \pm 4.17 \mathrm{AU})$ values in the control group increased significantly following the establishment of IMQ-induced psoriasis-like dermatitis over a period of 6 consecutive days (both $\mathrm{P}<0.05$ ). By contrast, the skin hydration value $(3.69 \pm 1.55 \mathrm{AU})$ of the control group decreased significantly on day 6 compared with that of day 0 $(\mathrm{P}<0.05$; Fig. 1C-E).

High-dose magnolol $(300 \mu \mathrm{g} / \mathrm{ml})$ treatment led to a restoration of $>30 \%$ of the TEWL value compared with the control and vehicle control groups on day 11 (both $\mathrm{P}<0.05$ ). The topical application of DXM markedly reduced the TEWL and erythema values compared with all the other groups on day 11 (all $\mathrm{P}<0.05$; Fig. 1C and D). However, magnolol treatment did not significantly affect erythema or skin hydration on psoriasis-like skin mice compared with the control and vehicle group on day 11 (all P>0.05; Fig. 1D and E).

DXM inhibits hyperproliferation of keratinocytes. The morphology of all 5 groups revealed the presence of scales, erythema and dry skin (Fig. 2A). The results of histopathology using $\mathrm{H} \& \mathrm{E}$ staining revealed epidermal acanthosis, parakeratosis, tortuous capillary dilatation in the papillary dermis, and the infiltration of various types of inflammatory cell in all groups (Fig. 2B). However, the DXM treatment group exhibited improved clinical and pathological features of the psoriasis severity compared with all other groups (Fig. 2A and B).

Magnolol activates the protein expression of PPAR- $\gamma$. To investigate whether magnolol acts as a PPAR- $\gamma$ agonist on the epidermis, PPAR- $\gamma$ protein expression levels were evaluated via immunohistochemical staining. The results demonstrated that magnolol activated PPAR- $\gamma$ protein expression on the epidermis, which was more clearly observed in the cytoplasm (Fig. 2C). Fig. 2D demonstrated the results of the immune-intensity analysis of PPAR- $\gamma$ via automatic computer counting. The high-dose magnolol treatment group exhibited higher immune-intensities of PPAR- $\gamma$ compared with all the other groups $(\mathrm{P}<0.05)$.

Magnolol inhibits the protein expression levels of IL-23, $I L-1 \beta, I L-6, T N F-\alpha$ and INF- $\gamma$ in psoriasis-like skin. The potential underlying mechanisms of magnolol treatment in psoriasis-like skin were examined using the inflammatory cytokine panel. The cytokine array results revealed that the expression of all the cytokines were significantly increased in the control group (IMQ-induced only) compared with the normalgroup (untreated skin specimens, $\mathrm{P}<0.05$ ). High-dose administration of magnolol led to the inhibition of IL-23, IL-1 $\beta$, IL-6, TNF- $\alpha$ and INF- $\gamma$ protein expression (all $\mathrm{P}<0.05$ ), although not of IL-17A ( $>>0.05)$, compared with the control 

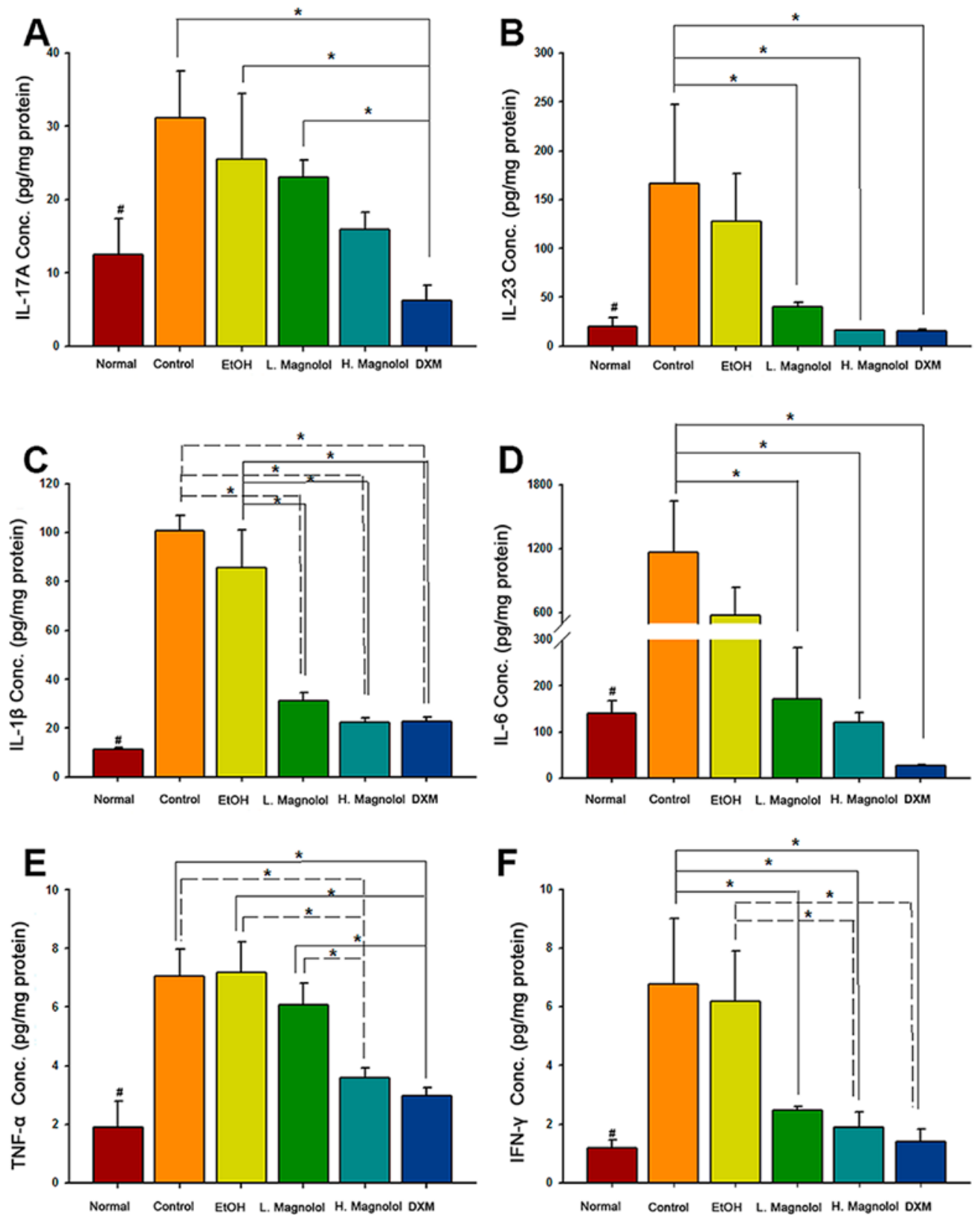

Figure 3. Magnolol inhibits the protein expression levels of IL-23, IL-1 $\beta$, IL-6, TNF- $\alpha$ and INF- $\gamma$ in psoriasis-like skin. (A) IL-17A, (B) IL-23, (C) IL-1 $\beta$, (D) IL-6, (E) TNF- $\alpha$ and (F) IFN- $\gamma$ protein expression levels were analyzed using a LEGENDplex ${ }^{\mathrm{TM}}$ kit (mean \pm standard deviation, $\mathrm{n}=3$ ); ${ }^{\sharp \mathrm{P}}<0.05$, compared with control group; " $\mathrm{P}<0.05$ (using two-way mixed ANOVA, post hoc Bonferroni's test). IL, interleukin; TNF- $\alpha$, tumor necrosis factor- $\alpha$; IFN- $\gamma$, interferon- $\gamma$.

group. Low-dose administration of magnolol led to the inhibition of IL-23, IL-1 $\beta$, IL-6 and INF- $\gamma$ protein expression (all $\mathrm{P}<0.05$ ) although not of IL-17A and TNF- $\alpha$ (both $\mathrm{P}>0.05$ ), compared with the control group. Both high and low administration of magnolol led to the inhibition of IL-1 $\beta$ compared with the EtOH group (both $\mathrm{P}<0.05$ ). Additionally, DXM inhibited the expression of all inflammatory cytokines, compared with the normal group $(\mathrm{P}<0.05$; Fig. 3$)$.

\section{Discussion}

The effect of magnolol on psoriasis has been rarely reported. The present study has demonstrated that magnolol activates PPAR- $\gamma$, and also significantly inhibits the protein expression of IL-23, IL-1 $\beta$, IL-6, TNF- $\alpha$ and INF- $\gamma$, which may contribute to skin barrier function. The IL-23/IL-17 axis has been reported to be a critical regulator for psoriasis and psoriatic arthritis (5). Previous studies have reported that IMQ-induced psoriasis-like skin elicits either protein or mRNA expression of IL-17, IL-23, IL-1 $\beta$, IL-6, TNF- $\alpha$ and INF- $\gamma$ in mice skin $(5,24-26)$, and successful anti-psoriatic interventions should therefore inhibit the aforementioned cytokine expression (24-26), especially with respect to the downregulation of IL-17 and IL-23 expression (23). However, in the present study, administration of high and low-dose magnolol treatment did not effectively inhibit IL-17 or lead to any improvement in the clinical and pathological features of the psoriasis severity index.

PPARs have been indicated to perform essential roles in cutaneous homeostasis (18). PPAR- $\gamma$, as one of three PPARs 
isoforms, has been indicated to exert anti-inflammatory effects on a variety of cell types, including macrophages, lymphocytes and connective tissue cells (27). PPAR $-\gamma$ has been reported to be localized mainly in the nucleus during a number of cellular processes (28). It was recently demonstrated that the downregulation of PPAR- $\gamma$ by its mitogen-activated protein kinase-dependent is redistributed from the nucleus to the cytosol for non-genomic activity $(28,29)$. A previous study demonstrated that PPAR- $\gamma$ mainly appears to localize in the cytoplasm in human keratinocytes, whereas it exhibits an exclusively nuclear localization in the suprabasal layer (30). The immunohistochemistry staining results presented in the current study exhibited a similar pattern. Therefore, PPAR- $\gamma$ may control the cytoplasmatic activity through the same mechanism in keratinocytes. An in vitro study revealed that PPAR- $\gamma$ regulated inflammatory signals by first inhibiting $\mathrm{NF}-\kappa \mathrm{B}$ nuclear translocation, and then downregulating the cytokine protein expression of IL-6, IL-8, IL-12, IL-21, IL-23, TNF- $\alpha$ and cyclooxygenase-2 (31). PPAR- $\gamma$ is expressed in keratinocytes, and is also involved in the regulation of keratinocyte differentiation (18). Thiazolidinediones, a family of PPAR- $\gamma$ ligands, have been indicated to reduce epidermal keratinocyte proliferation and promote epidermal keratinocyte differentiation in a repeated tape stripping-induced hyperproliferative animal model (18). These results may suggest that topical PPAR- $\gamma$ agonists could be considered as a potential adjunctive therapeutic agent in hyperproliferative skin diseases, such as psoriasis (32).

The bark of Magnolia officinalis has been traditionally used in Asia for the treatment of a number of diseases, including anxiety, asthma and depression (33). Honokiol is another primary active compound that is isolated from Magnolia officinalis, and is also a PPAR- $\gamma$ agonist (15-17). A previous study revealed that honokiol could effectively improve psoriasis treatment by inhibiting the $\mathrm{NF}-\kappa \mathrm{B}$ pathway in a transgenic mouse model (34). A previous study also indicated the effect and mechanism of magnolol on psoriasis mice induced by imiquimod via oral administration (35). To the best of our knowledge, the present study has been the first to demonstrate the topical anti-psoriatic effects as well as skin barrier function improvement of magnolol on IMQ-induced psoriasis-like dermatitis in mice.

The number of animals used in the current study was small $(n=3)$. In future studies, the number of animals used should be increased. Further research is also required to enhance the topical distribution of magnolol to the skin and to investigate the therapeutic outcome of this treatment, as well as to clarify the pharmacological effects of magnolol on psoriasis.

The IL-23/IL-17 axis has been reported to be the critical regulator for psoriasis and psoriatic arthritis $(5,6)$. In the present study, it has been demonstrated that magnolol activates PPAR- $\gamma$, and is able to improve barrier function via downregulation of the IL-23 signaling pathway in an IMQ-induced psoriasis-like dermatitis mouse model. The results revealed that the downregulation of IL-23 may contribute to barrier function improvement, and could possibly serve a role in alleviating psoriasis-like dermatitis in animals. However, the application of magnolol alone, when applied topically to inhibit IL-23, may not be an effective method for psoriasis treatment. The potential of using systemic magnolol or topical magnolol treatment combined with topical glucocorticosteroid to treat psoriasis, however, is worth of further investigation.

\section{Acknowledgements}

The authors would like to thank Dr Tsen-Fang, Tsai from National Taiwan University Hospital, who provided insights and expertise that greatly assisted the research project with regards to the animal study design.

\section{Funding}

The present study was supported by grants from the Ministry of Science and Technology of Taiwan (grant nos. MOST-1072314-B-281-010- and MOST-108-2314-B-281-002-) and from Cathay General Hospital (grant nos. CGH-MR-A106024 and CGH-MR-A109017).

\section{Availability of data and materials}

The datasets used and/or analyzed during the current study are available from the corresponding author on reasonable request.

\section{Authors' contributions}

The study was conceptualized and designed by JWG, YPC, and SHJ. JWG wrote the initial version of the manuscript. JWG, YO, and CYW performed the animal studies and barrier function study. YPC and SHJ interpreted the animal image data regarding the severity of psoriasis. JWG and CYL performed the immunostaining and CYL performed the pathologic diagnosis. JWG performed the cytokines array and statistical analyses. HYT was responsible for reviewing and editing the paper. JWG, YPC, and SHJ provided supervision throughout the study. All authors read and approved the final manuscript.

\section{Ethics approval and consent to participate}

All animal experiments were performed and approved by the Institutional Animal Care and Use Committee (IACUC) of Cathay General Hospital (IACUC registration no. 107-028).

\section{Patient consent for publication}

Not applicable.

\section{Competing interests}

The authors declare that they have no competing interests.

\section{References}

1. Elias PM, Hatano Y and Williams ML: Basis for the barrier abnormality in atopic dermatitis: Outside-inside-outside pathogenic mechanisms. J Allergy Clin Immunol 121: 1337-1343, 2008.

2. Azfar RS and Gelfand JM: Psoriasis and metabolic disease: Epidemiology and pathophysiology. Curr Opin Rheumatol 20: 416-422, 2008

3. Gudjonsson JE and Elder JT: Psoriasis. In: Fitzpatrick's Dermatology in General Medicine. Goldsmith LA, Kazt SI, Gilchrest BA, Paller AD, Leffell DJ and Wolff K (eds.). McGraw-Hill Companies, Inc., 2012. 
4. Boehncke WH and Schon MP: Psoriasis. Lancet 386: 983-994, 2015.

5. Di Cesare A, Di Meglio P and Nestle FO: The IL-23/Th17 axis in the immunopathogenesis of psoriasis. J Invest Dermatol 129: $1339-1350,2009$.

6. Toussirot E: The IL23/Th17 pathway as a therapeutic target in chronic inflammatory diseases. Inflamm Allergy Drug Targets 11: 159-168, 2012.

7. Bowcock AM: The genetics of psoriasis and autoimmunity. Annu Rev Genomics Hum Genet 6: 93-122, 2005

8. Alonso-Castro AJ, Zapata-Bustos R, Dominguez F, Garcia-Carranca A and Salazar-Olivo LA: Magnolia dealbata Zucc and its active principles honokiol and magnolol stimulate glucose uptake in murine and human adipocytes using the insulin-signaling pathway. Phytomedicine 18: 926-933, 2011.

9. Dominguez F, Chavez M, Garduno-Ramirez ML Chavez-Avila VM, Mata M and Cruz-Sosa F: Honokiol and magnolol production by in vitro micropropagated plants of Magnolia dealbata, an endangered endemic Mexican species. Nat Prod Commun 5: 235-240, 2010.

10. Kuo DH, Lai YS, Lo CY, Cheng AC, Wu H and Pan MH: Inhibitory effect of magnolol on TPA-induced skin inflammation and tumor promotion in mice. J Agric Food Chem 58: 5777-5783, 2010.

11. Lee YJ, Lee YM, Lee CK, Jung JK, Han SB and Hong JT: Therapeutic applications of compounds in the Magnolia family. Pharmacol Ther 130: 157-176, 2011.

12. Lin CF, Hwang TL, Al-Suwayeh SA, Huang YL, Hung YY and Fang JY: Maximizing dermal targeting and minimizing transdermal penetration by magnolol/honokiol methoxylation. Int J Pharm 445: 153-162, 2013.

13. Shen JL, Man KM, Huang PH, Chen WC, Chen DC, Cheng YW, Liu PL, Chou MC and Chen YH: Honokiol and magnolol as multifunctional antioxidative molecules for dermatologic disorders. Molecules 15: 6452-6465, 2010.

14. Tanaka K, Hasegawa J, Asamitsu K and Okamoto T: Magnolia ovovata extract and its active component magnolol prevent skin photoaging via inhibition of nuclear factor kappaB. Eur J Pharmacol 565: 212-219, 2007.

15. Dreier D, Latkolik S, Rycek L, Schnürch M, Dymáková A, Atanasov AG, Ladurner A, Heiss EH, Stuppner $H$, Schuster D, et al: Linked magnolol dimer as a selective PPARg agonist-Structure-based rational design, synthesis, and bioactivity evaluation. Sci Rep 7: 13002, 2017.

16. Wang L, Waltenberger B, Pferschy-Wenzig EM, Blunder M, Liu X, Malainer C, Blazevic T, Schwaiger S, Rollinger JM, Heiss EH, et al: Natural product agonists of peroxisome proliferator-activated receptor gamma (PPARg): A review. Biochem Pharmacol 92: 73-89, 2014

17. Dreier D, Resetar M, Temml V, Rycek L, Kratena N, Schnürch M, Schuster D, Dirsch VM and Mihovilovic MD: Magnolol dimer-derived fragments as PPARg-selective probes. Org Biomol Chem 16: 7019-7028, 2018.

18. Mao-Qiang M, Fowler AJ, Schmuth M, Lau P, Chang S, Brown BE, Moser AH, Michalik L, Desvergne B, Wahli W, et al: Peroxisome-proliferator-activated receptor (PPAR)-gamma activation stimulates keratinocyte differentiation. J Invest Dermatol 123: 305-312, 2004.

19. Flori E, Mastrofrancesco A, Kovacs D, Bellei B, Briganti S, Maresca V, Cardinali G and Picardo M: The activation of PPARg by 2,4,6-Octatrienoic acid protects human keratinocytes from UVR-induced damages. Sci Rep 7: 9241, 2017.

20. Horvath S, Komlodi R, Perkecz A, Pinter E, Gyulai R and Kemeny A: Methodological refinement of Aldara-induced psoriasiform dermatitis model in mice. Sci Rep 9: 3685, 2019.

21. Chong HT, Yang GN, Sidhu S, Ibbetson J, Kopecki Z and Cowin AJ: Reducing Flightless I expression decreases severity of psoriasis in an imiquimod-induced murine model of psoriasiform dermatitis. Br J Dermatol 176: 705-712, 2017.
22. van der Fits L, Mourits S, Voerman JS, Kant M, Boon L, Laman JD, Cornelissen F, Mus AM, Florencia E, Prens EP and Lubberts E: Imiquimod-induced psoriasis-like skin inflammation in mice is mediated via the IL-23/IL-17 axis. J Immunol 182 5836-5845, 2009.

23. Guo JW, Cheng YP, Liu CY, Thong HY, Huang CJ, Lo Y, Wu CY and Jee SH: Salvianolic acid B in microemulsion formulation provided sufficient hydration for dry skin and ameliorated the severity of imiquimod-induced psoriasis-like dermatitis in mice. Pharmaceutics 12: 457, 2020.

24. Lin ZM, Ma M, Li H, Qi Q, Liu YT, Yan YX, Shen YF, Yang XQ, Zhu FH, He SJ, et al: Topical administration of reversible SAHH inhibitor ameliorates imiquimod-induced psoriasis-like skin lesions in mice via suppression of TNF- $\alpha / \mathrm{IFN}-\mathrm{g}$-induced inflammatory response in keratinocytes and T cell-derived IL-17. Pharmacol Res 129: 443-452, 2018.

25. Li YL, Du ZY, Li PH, Yan L, Zhou W, Tang YD, Liu GR, Fang YX, Zhang K, Dong CZ and Chen HX: Aromatic-turmerone ameliorates imiquimod-induced psoriasis-like inflammation of BALB/c mice. Int Immunopharmacol 64: 319-325, 2018.

26. OuYang Q, Pan Y, Luo H, Xuan C, Liu J and Liu J: MAD ointment ameliorates imiquimod-induced psoriasiform dermatitis by inhibiting the IL-23/IL-17 axis in mice. Int Immunopharmacol 39: 369-376, 2016

27. Ramot Y, Mastrofrancesco A, Camera E, Desreumaux P, Paus R and Picardo M: The role of PPAR $\gamma$-mediated signalling in skin biology and pathology: New targets and opportunities for clinical dermatology. Exp Dermatol 24: 245-251, 2015.

28. Burgermeister E and Seger R: MAPK kinases as nucleo-cytoplasmic shuttles for PPARgamma. Cell Cycle 6: 1539-1548, 2007.

29. Burgermeister E, Chuderland D, Hanoch T, Meyer M, Liscovitch M and Seger R: Interaction with MEK causes nuclear export and downregulation of peroxisome proliferator-activated receptor gamma. Mol Cell Biol 27: 803-817, 2007.

30. Westergaard M, Henningsen J, Svendsen ML, Johansen C, Jensen UB, Schrøder HD, Kratchmarova I, Berge RK, Iversen L, Bolund L, et al: Modulation of keratinocyte gene expression and differentiation by PPAR-selective ligands and tetradecylthioacetic acid. J Invest Dermatol 116: 702-712, 2001.

31. Mastrofrancesco A, Kovacs D, Sarra M, Bastonini E, Cardinali G Aspite N, Camera E, Chavatte P, Desreumaux P, Monteleone G and Picardo M: Preclinical studies of a specific PPARg modulator in the control of skin inflammation. J Invest Dermatol 134: 1001-1011, 2014

32. Demerjian M, Man MQ, Choi EH, Brown BE, Crumrine D, Chang S, Mauro T, Elias PM and Feingold KR: Topical treatment with thiazolidinediones, activators of peroxisome proliferator-activated receptor-gamma, normalizes epidermal homeostasis in a murine hyperproliferative disease model. Exp Dermatol 15: 154-160, 2006.

33. Poivre $\mathrm{M}$ and Duez P: Biological activity and toxicity of the Chinese herb magnolia officinalis rehder \& E. Wilson (Houpo) and its constituents. J Zhejiang Univ Sci B 18: 194-214, 2017.

34. Wen J, Wang X, Pei H, Xie C, QiuN, Li S, Wang W, Cheng X and Chen L: Anti-psoriatic effects of Honokiol through the inhibition of NF-kappaB and VEGFR-2 in animal model of K14-VEGF transgenic mouse. J Pharmacol Sci 128: 116-124, 2015.

35. Tsai ZX, Mo SY and Zhu SG: Effect and mechanism of Magnolol on psoriasis mice induced by imiquimod. Chin J Clin Pharmacol 35: 56-59, 2019 (In Chinese).

This work is licensed under a Creative Commons Attribution-NonCommercial-NoDerivatives 4.0 International (CC BY-NC-ND 4.0) License. 